\title{
Criminologie
}

\section{Éditorial : la dimension comparative}

\section{Maurice Cusson}

Volume 26, numéro 2, 1993

La criminologie comparée. Hommage à Denis Szabo

URI : https://id.erudit.org/iderudit/017335ar

DOI : https://doi.org/10.7202/017335ar

Aller au sommaire du numéro

Éditeur(s)

Les Presses de l'Université de Montréal

ISSN

0316-0041 (imprimé)

1492-1367 (numérique)

Découvrir la revue

Citer ce document

Cusson, M. (1993). Éditorial : la dimension comparative. Criminologie, 26(2),

7-12. https://doi.org/10.7202/017335ar d'utilisation que vous pouvez consulter en ligne.

https://apropos.erudit.org/fr/usagers/politique-dutilisation/ 
LA DIMENSION COMPARATIVE

Maurice Cusson ${ }^{1}$

Denis Szabo nous apporte des nouvelles de l'étranger. Il nous met en contact avec les criminologues d'ailleurs. Il fait entrer le vent du large à l'École de criminologie et au Centre international de criminologie comparée. Cette confrontation avec les criminologues d'ailleurs, cette volonté de nous situer d'emblée dans le club des meilleurs, voilà, pour Denis Szabo, la criminologie comparée en action. Il la comprend comme seul un citoyen du monde peut la comprendre. Il sait, parce qu'il l'a vu, que les problèmes criminels se posent sensiblement en les mêmes termes un peu partout en Occident et peut-être mềme au-delà. Avec plusieurs, il pense que la terre est devenue un village global.

Contre les sceptiques et les relativistes avec lesquels il entretient une relation d'amour/haine, Denis Szabo persiste et signe : la nature humaine existe bel et bien. Au-delà de l'accessoire et du surajouté, il existe un substrat permanent, naturel à l'être humain (Szabo, $1978:$ p. 63). Cela le conduit à poser la question criminelle en des termes universaux : «La vie en société exige des règles; la liberté de l'homme implique la possibilité de leur transgression $»(1986:$ p. 12-13). C'est en prenant appui sur l'universalité de la condition humaine, de la norme, de l'infraction et de la sanction qu'il se lance dans l'aventure comparatiste. La comparaison n'est possible que sur une toile de fond constante. Si la criminalité des divers pays du globe se réduisait à un agrégat de constructions purement arbitraires et totalement relatives, le jeu des comparaisons n'aurait pas de sens. Comparer le comparable, c'est confronter des entités de même espece. La criminologie comparée n'est donc possible que parce qu'on s'entend sur la nature du crime au sein de l'aire où la comparaison est faite. La criminologie comparée n'a que faire de l'exotisme.

\section{LE TOUT COMPARATIF}

Le lecteur cherche en vain une définition explicite de la criminologie comparée sous la plume de Denis Szabo. Ce n'est pas par hasard : notre fondateur a toujours refusé de se laisser enfermer dans un système qui limiterait sa marge de manœuvre. Une définition impose inévitablement des critères

1. Professeur et directeur de l'École de criminologie de l'Université de Montréal, C.P. 6128, Succ. * A \%, Montréal (Québec) H3C 3J7. 
d'inclusion et d'exclusion. Son aecuménisme l'a empêché d'en venir à cette extrêmité.

De toute manière, il est loisible à Denis Szabo de penser que l'esprit humain procède presque toujours par comparaison et d'en conclure que toute bonne criminologie est nécessairement comparative. C'est indiscutable : nous comparons des délinquants et des non-délinquants; des conformistes culturels et des manipulateurs; des psychopathes et des psychotiques. Quand nous disons que telle peine est sévère, nous sous-entendons qu'elle l'est moins que telle autre qui a êté infligée à un individu ayant commis un crime semblable à celui qui est sanctionné. Dire d'une corrélation qu'elle est faible, c'est dire qu'elle l'est relativement à une autre. Le thème de l'augmentation de la criminalité est comparatif : on compare les taux de l'année qui vient de s'écouler avec ceux des années antérieures. Pourquoi dit-on que les Américains et les Canadiens sont punitifs? Parce qu'on pense aux taux d'incarcération des Hollandais.

Bref, nous faisons de la criminologie comparée comme M. Jourdain faisait de la prose : sans le savoir. Mais si tout est comparatif, le manteau de la criminologie comparée s'étend à toute la criminologie et il n'y a plus moyen de distinguer l'une de l'autre.

\section{LA DÉMARCHE COMPARATIVE}

Si on se donne la peine de parler de criminologie comparée, ce n'est pas seulement comme d'un synonyme de criminologie. On veut être plus spécifique. On veut communiquer l'idée des variations transculturelles, l'idée que la question criminelle se pose de manière à la fois semblable et différente dans divers ensembles sociaux d'importance. Mais il existe très peu de publications en criminologie dont la comparaison à l'échelle macroscopique soit le premier objectif. En revanche, on trouve de nombreux travaux qui ont une dimension comparative. Cette dernière intervient à titre de moyen, dans une démarche dont le but principal est autre.

Si la criminologie comparée en tant que telle est rare, la démarche comparative l'est beaucoup moins. Dans ce cas, il importe de préciser en quoi elle consiste. Tout d'abord, une définition.

En criminologie la démarche comparative consiste, en premier lieu, à examiner les ressemblances et les différences entre les crimes, les criminels, la criminalité et la réaction sociale dans au moins deux ensembles sociaux ou au moins à deux moments différents au sein d'un même ensemble social. Elle consiste, en second lieu, à déterminer l'extension d'une théorie criminologique en vérifiant si elle vaut dans plus d'un ensemble social ou à des époques différentes. 
Par ensemble social, j'entends des unités sociologiques relativement vastes : pays, provinces, villes... C'est donc dire que la démarche comparative porte sur les variations spatio-temporelles macroscopiques de tout ce qui se rapporte au phénomène criminel : criminalité, taux d'incarcération, taux de solution de divers crimes, caractéristiques des criminels...

\section{LA GLOBALISATION DES PROBLÈMES CRIMINELS}

La démarche comparative s'impose de plus en plus parce que les problèmes criminels se posent en des termes de plus en plus semblables partout dans notre aire de civilisation. L'intensification des échanges internationaux de biens, de services, de personnes et d'idées produit de puissants courants qui traversent la planète dans la plupart des secteurs de l'activité humaine : économie, démographie, politique, éthíque... Notre domaine n'y échappe pas. Ainsi, la criminalité présente la même allure dans presque tous les pays occidentaux. Partout les délits contre les biens s'imposent par leur fréquence et les crimes violents dépassent rarement $10 \%$ de l'ensemble des crimes enregistrés par la police. Pratiquement partout, les délits les plus fréquents sont des actes furtifs ou expéditifs; leurs auteurs utilisent des tactiques rudimentaires et ils n'en tirent que des bénéfices médiocres (Cusson, 1993). Les crimes qui sont bien mesurés par les statistiques policières ont connu une augmentation fulgurante dans presque tous les pays occidentaux entre 1960 et 1975 et tendent à plafonner depuis. L'augmentation parallèle du trafic et de la consommation de la drogue exerce une influence sensible sur la délinquance conventionnelle. Tout cela, un criminologue québécois a intérêt à le savoir, sinon, il risquerait, en se limitant à la criminalité du Québec, d'attribuer à des caractéristiques locales ce qui relève en fait d'une évolution que connaît toute notre aire de civilisation.

Il est indispensable de se donner des points de comparaison si l'on prétend poser un bon diagnostic macrocriminologique (estimation de l'ampleur du problème criminel qui se pose dans une juridiction donnée, description des caractéristiques de la criminalité locale, identification des patterns criminels les plus préoccupants, localisation des endroits où l'activité criminelle est la plus virulente...). L'analyse des tendances de la criminalité permet de comparer les crimes du présent à ceux du passé et de vérifier si les problèmes s'aggravent ou non. L'examen de la criminalité dans les unités géographiques voisines permet d'établir le degré de spécificité des problèmes locaux. La connaissance des crimes commis ailleurs et durant les années passées s'impose à l'observateur qui désire obtenir une vision juste de la conjoncture criminelle qu'il analyse.

Les Occidentaux réagissent au crime selon des patterns de plus en plus communs. Les sondages sur les perceptions de la gravité de divers types de 
crimes démontrent que le public attribue un ordre de gravité étonnamment semblable d'un pays à l'autre (Normandeau, 1970). Dans le domaine correctionnel, les tendances convergentes ne manquent pas. Notons la croissance des peines non carcérales et la diminution de la certitude de l'incarcération (entre 1960 et 1975, la criminalité ayant augmenté beaucoup plus rapidement que le nombre d'individus incarcérés, les pourcentages des crimes commis effectivement sanctionnés ont eu tendance à décroître) (Cusson, 1990).

Un comparatiste de haut vol, D. Bayley (1992), a montré que l'organisation et les tactiques policières sont coulées dans le même moule dans quatre pays où la langue anglaise domine. En effet, en Australie, au Canada, aux États-Unis et en Grande-Bretagne, on trouve dans la plupart des corps policiers trois départements spécialisés comptant sensiblement les mêmes pourcentages d'effectifs :

1. Les opérations, aussi appelées les services généraux et la patrouille : les policiers affectés à ce département répondent aux appels des citoyens; ils patrouillent; ils répondent aux urgences et maintiennent l'ordre; ils représentent environ $60 \%$ des effectifs de la plupart des corps policiers.

2. Les enquêtes criminelles, attribuées à des détectives (10 à $15 \%$ des effectifs).

3. Le trafic (la circulation dans les rues) : (10\% des effectifs).

Les trois principales tactiques de lutte contre le crime que l'on trouve dans les forces de police de ces quatre pays sont : premièrement, la réponse rapide aux appels dans le but d'attraper des délinquants sur le fait; deuxièmement, la patrouille; troisièmement, l'enquête criminelle.

Bayley fait observer que cee modele de fonctionnement est apparu sensiblement à la même époque au tout début du XX⿳亠丷厂 $\mathrm{X}^{\mathrm{c}}$ siècle - en Angleterre, en Australie, au Canada et aux États-Unis. Selon lui, ces similitudes s'expliquent par un processus rapide d'imitations et d'emprunts. Les spécialistes des questions policières (œuvrant souvent dans des commissions) étudient les solutions adoptées ailleurs avant de proposer leur propre modèle. Vers la fin du XIX ${ }^{\mathrm{e}}$ siècle émergea l'idée selon laquelle les enquêtes criminelles devraient être distinguées des autres opérations policières et que l'organisation des corps de police devrait être réaménagée en conséquence. Cette conception s'est diffusée très rapidement dans les pays de langue anglaise à la faveur des voyages, des congrès, de la circulation des écrits, etc. De nos jours, la police communautaire et le problem-oriented policing sont devenus à la mode dans les cercles internationaux réunissant les 
experts des questions policières. Bayley laisse entendre que l'histoire pourrait fort bien se répéter.

\section{DES THÉORIES UNIVERSELLES}

Les sciences sociales ne visent pas seulement à produire des connaissances qui prévaudraient partout et toujours. Il existe bel et bien une science des singularités sociales (Boudon, 1992). Il n'en reste pas moins que l'universalité est une visée majeure de nos sciences. En criminologie, l'univers du discours déborde régulièrement les frontières nationales. Nous parlons de la théorie de la personnalité criminelle comme d'un modèle qui rend compte du fonctionnement d'une catégorie universelle de délinquants, et pas seulement des délinquants québécois. Nous raisonnons sur les facteurs de la criminalité comme s'ils produisaient partout les mêmes effets. Nous présumons que les théories et les mesures qui prévalent aux ÉtatsUnis devraient prévaloir chez nous. De fait, l'existence de variables universellement associées au crime est attestée ; pensons à l'âge et au sexe. Une recherche menée dans 110 pays par Archer et Gartner (1976) démontre que, dans tous les pays qui ont connu une guerre, les taux d'homicides augmentent après la guerre. Certaines théories résistent remarquablement bien aux vérifications transculturelles. C'est ainsi que la théorie du contrôle social de Hirschi (1969) a subi avec succès le test de la vérification au Canada (Le Blanc, 1986), en Hollande (Junger-Tas, 1988) et en Angleterre (Riley et Shaw, 1985).

Récemment, Gartner (1990) a voulu identifier les variables associées à des taux d'homicides désagrégés selon l'âge et le sexe de la victime dans 18 pays développés entre 1950 et 1980 . Elle constate que les facteurs structurels et culturels en rapport avec les homicides aux États-Unis, par exemple le divorce, le travail des femmes et l'inégalité, sont aussi à l'œuvre à l'échelle internationale. Les variations internationales et les variations annuelles (entre 1950 et 1980) des taux agrégés d'homicides sont positivement et significativement associés aux taux de divorces et à l'indice GINI d'inégalité. Ils sont négativement et significativement associés aux niveaux de dépenses d'aide sociale. S'agissant des taux d'homicides ayant une fermme pour victime, ils varient en raison directe du nombre de femmes qui travaillent hors de la maison.

Malheureusement, nos visées universalistes se heurtent rapidement aux limites des particularismes et des configurations singulières. L'exemple du Japon est bien connu. Dans pratiquement tous les pays développés, la croissance économique de l'après-guerre s'accompagna d'une augmentation de la criminalité, mais cela n'est pas vrai au Japon (Bayley, 1976; Cusson, 1990). Comparant les homicides commis aux États-Unis et en Suède, Wikstrom 
(1992) fait observer qu'aux États-Unis les armes à feu, la race et les ghettos sont au cœur des explications proposées pour rendre compte de la distribution des homicides. Or, en Suede, aucun de ces thèmes n'a de l'importance dans les hypothèses avancées. Celles qui reviennent le plus souvent mettent en cause l'alcool, l'immigration et la marginalité (les meurtres suédois impliquent souvent des toxicomanes et des criminels).

Bref, la démarche comparative s'impose de plus en plus parce que la criminalité présente des caractéristiques communes dans la plupart des démocraties occidentales, parce qu'il existe des tendances communes dans la réaction sociale du crime, que les institutions policières et correctionnelles se ressemblent et que la démarche comparative est un moment indispensable d'une recherche théorique qui entend spécifier la portée et la limite des généralisations proposées.

\section{BIBLIOGRAPHIE}

ARCHER, D. et R. GARTNER (1984), Violence and Crime in Cross-National Perspective, New Haven, Yale University Press.

BAlLEY, D.H. (1976), Forces of Order. Police Behavior in Japan and The United States, Berkeley, University of California Press.

BAILEY, D.H. (1976), "Comparative Organization of the Police in English speaking Countries $\%$, in M. TONRY et N. MORRIS (éds), Modern Policing. Crime and Justice, vol. 15, Chicago, University of Chicago Press, p. 509-546.

BOUDON, R. (1992), «Introduction», in R. BOUDON (๕d), Traité de sociologie, Paris, PUF, p. 5-20.

CUSSON, M. (1990), Croissance et décroissance du crime, Paris, PUF.

CUSSON, M. (1993), «A Strategic analysis of crime : criminal tactics as responses to precriminal situations ", Advances in Criminological Theory.

GARTNER, R. (1990), *The Victims of Homicide : A Temporal and Cross-national Comparaison », American Sociological Review, vol. 55, p. 92-106.

HIRSCHI, T. (1969), Causes of delinquency, Berkeley, University of California Press.

JUNGER-TAS, J. (1988), «Causal factors : social control theory *, in I. JUNGER-TAS et R.L. BLOCK, (éds), Juvenile delinquency in the Netherland, Amstelveen, Kugler.

LE BLANC, M. (1986), « Pour une approche intégrative de la conduite délinquante des adolescents $*$, Criminologie, vol. XIX, $\mathrm{n}^{\circ} 1$, p. 73-96.

NORMANDEAU, A. (1970), «Etude comparative d'un indice pondéré de la criminalité dans 8 pays ", Revue International de Police criminelle, p. 15-18.

RILEY, D. et M. SHAW (1985), Parental Supervision and Juvenile Delinquency, Home Office Research Study, $n^{\circ} 83$, London.

SZABO, D. (1978), Criminologie et politique criminelle, Montréal, PUM/Paris, J. Vrin. WIKSTRÖM, P.-O. H. (1992), « Cross-National Comparisons and context-specific trends in criminal homicide », Journal of Crime and Justice, vol. XIV, $\mathrm{n}^{\circ}$ 2, p. 1-25. 